\title{
Phenoxythiophene sulfonamide compound B355252 protects neuronal cells against glutamate-induced excitotoxicity by attenuating mitochondrial fission and the nuclear translocation of AIF
}

\author{
YUXIN ZHANG $^{1-3}$, NAILYA S. GLIYAZOVA ${ }^{1}$, P. ANDY LI $^{1}$ and GORDON IBEANU ${ }^{1}$ \\ ${ }^{1}$ Department of Pharmaceutical Sciences, Biomanufacturing Research Institute and Technology Enterprise (BRITE), \\ North Carolina Central University, Durham, NC 27707, USA; ${ }^{2}$ Institute of Clinical Pharmacology, \\ Department of Pharmacy, General Hospital of Ningxia Medical University; ${ }^{3}$ School of Pharmacy, \\ Ningxia Medical University, Yinchuan, Ningxia 750004, P.R. China
}

Received June 3, 2020; Accepted December 3, 2020

DOI: $10.3892 / \mathrm{etm} .2021 .9652$

\begin{abstract}
Glutamate neurotoxicity has been implicated in the initiation and progression of various neurological and neurodegenerative disorders. Therefore, it is necessary to develop therapeutics for the treatment of patients with these devastating diseases. Mitochondrial fission plays an import role in the mediation of cell death and survival. The objective of the present study was to determine whether B355252, a phenoxythiophene sulfonamide derivative, reduces glutamateinduced cell death by inhibiting mitochondrial fission and the nuclear translocation of apoptosis-inducing factor (AIF) in glutamate-challenged HT22 neuronal cells. The results revealed that glutamate treatment led to large increases in the mitochondrial levels of the major fission proteins dynamin-related protein 1 (Drpl) and mitochondrial fission 1 protein (Fis1), but only small elevations in the fusion proteins mitofusin 1 and 2 (Mfn1/2) and optic atrophy 1 (Opa1). In addition, glutamate toxicity disrupted mitochondrial reticular networks and increased the translocation of AIF to the nucleus. Pretreatment with B35525 reduced glutamate-induced cell death and prevented the increases in the protein levels of Drp1, Fis1, Mfn1/2 and Opa1 in the mitochondrial fraction. More importantly, the architecture of the mitochondria was
\end{abstract}

Correspondence to: Dr Gordon Ibeanu or Dr P. Andy Li, Department of Pharmaceutical Sciences, Biomanufacturing Research Institute and Technology Enterprise (BRITE), North Carolina Central University, BRITE Building 1017, 302 East Lawson Street, Durham, NC 27707, USA

E-mail: gibeanu@nccu.edu

E-mail: pli@nccu.edu

Key words: B355252, arylthiophene, phenoxythiophene, glutamate, neuronal cell death, mitochondrial fission, mitochondria dynamics, apoptosis-inducing factor protected and nuclear translocation of AIF was completely inhibited by B35525. These findings suggest that the regulation of mitochondrial dynamics is central to the neuroprotective properties of B355252, and presents an attractive opportunity for potential development as a therapy for neurodegenerative disorders associated with mitochondria dysfunction.

\section{Introduction}

Glutamate is a major mediator of excitatory neurotransmission in the mammalian central nervous system (CNS), and plays crucial roles in the physiology of numerous CNS functions ranging from learning to memory and cognition (1). The tonic concentrations of glutamate are tightly regulated in the synapse by high-affinity glutamate reuptake systems (2). However, high concentrations of glutamate in the CNS are potentially neurotoxic. The neurotoxic effects are generally characterized by neuronal hyperexcitability and excitotoxicity. Glutamate-induced excitotoxicity has been associated with inflammation and oxidative stress, and has emerged as an important pathological mechanism in a wide range of acute and chronic neurological disorders, including cerebral ischemia, epilepsy, traumatic brain injury and neurodegenerative diseases such as Alzheimer's disease, Parkinson's disease and Huntington's disease (3-6). The physiological basis for glutamate-induced neurotoxicity is beginning to emerge, but there is considerable uncertainty surrounding the underlying mechanisms. Several lines of evidence point to mitochondrial dysfunction as a defining event in glutamate-induced neurotoxicity. Mitochondria-dependent calcium influx and the depletion of the intracellular antioxidant glutathione promote acute oxidative stress by elevating the levels of reactive oxygen species (ROS), which in turn leads to an imbalance in mitochondrial dynamics and, ultimately, cell death (7).

Mitochondria are bioenergetic and biosynthetic organelles with a well-recognized role in the production of ATP and other macromolecules. It has become apparent that 
mitochondria perform various signaling functions, as evidence has emerged on their crucial role in the maintenance of intracellular calcium $\left[\mathrm{Ca}^{2+}\right]_{\mathrm{i}}$ homeostasis, regulation of the intracellular level of ROS and, most importantly, programmed cell death $(8,9)$. Mitochondria are highly dynamic intracellular entities that undergo biogenesis, clearance and fission/fusion. In these mitochondrial dynamics, the continuous cycles of fission and fusion are maintained in a delicate balance and are essential for mitochondrial homeostasis $(10,11)$. Fission and fusion events are tightly and coordinately regulated by several nuclear-encoded proteins with GTPase hydrolysis activities. Among them, dynamin-related protein 1 (Drp1) and mitochondrial fission 1 protein (Fis1) mediate mitochondrial fission, while mitofusin 1 and 2 (Mfn1/2) and optic atrophy 1 (Opa1) proteins mediate mitochondrial fusion (12). Drp1, the master regulator of mitochondrial fission, exists predominantly in the cytoplasm where, when being activated, it interacts with GTP and lipids to form oligomers that are subsequently recruited to the mitochondrial outer membrane to form an integral part of the fission machinery (13). Fis1 is a component protein of the fission complex that includes mitochondrial fission factor (Mff), mitochondrial division protein 1, and mitochondrial dynamics proteins of $49 \mathrm{kDa}$ (MiD49) and $51 \mathrm{kDa}$ (MiD51). Fis1 is anchored in the outer membrane of the mitochondria where it mediates the recruitment of oligomerized Drp1 to fission sites on the membrane. The translocation of Drp1 to the mitochondrial membrane initiates a series of events that culminates in the division of the mitochondria or the activation of apoptosis, depending on the triggering stimulus $(10,11,13)$. In contrast to mitochondrial fission, the process of mitochondrial fusion is positively regulated by Mfn1/2 and Opa1, and occurs in two phases. In the first phase, the dimerization and binding of mitofusins facilitates the tethering of the outer membranes of neighboring mitochondria via a process that involves lipid hydrolysis. In the second phase, Opal coordinates the formation of cristae and fusion of the mitochondrial inner membranes, thus completing the fusion process (12). The view that mitochondrial fusion is a requirement for the transport of mitochondria within the cell and essential for cell survival is commonly accepted.

The small molecule 4-chloro-N-(naphthalen-1-ylmethyl)-5[3-(piperazin-1-yl)phenoxy]thiophene-2-sulfonamide (B355252) is an pheneoxythiophene sulfonamide with intrinsic nerve growth factor (NGF) potentiating activity. B35525 has been shown to enhance the ability of NGF-primed NS-1 pheocromocytoma cells to differentiate into a neuron-like phenotype with extensive networks of branched neurites $(14,15)$. B35525 does not induce neurite outgrowth alone, but enhances the effect of sub-physiological concentrations of NGF on neurons in vitro. In addition, B355252 has exhibited unique activity in several in vitro models of chronic neurological and neurodegenerative disorders $(16,17)$. In our previous study, B355252 was shown to protect HT22 neuronal cell against glutamate-induced excitotoxicity via the potent suppression of the oxidative injury caused by ROS production and $\left[\mathrm{Ca}^{2+}\right]_{\mathrm{i}}$ overload (16). Given that mitochondria are the major site of $\left[\mathrm{Ca}^{2+}\right]_{\mathrm{i}}$ and ROS production during glutamate excitotoxicity, the present study examined the effect of B355252 on HT22 cell viability and markers of mitochondrial structural dynamics and apoptosis following glutamate exposure.

\section{Materials and methods}

Cell culture and experimental treatment. HT22 murine hippocampal neuronal cells (donated by Dr June Panee; University of Hawaii, Honolulu, Hawaii) were cultured in Dulbecco's Modified Eagle's Medium (DMEM; Invitrogen; Thermo Fisher Scientific, Inc.) supplemented with $10 \%$ heat-inactivated fetal bovine serum (FBS; cat. no. MT35011CV; Thermo Fisher Scientific, Inc.), 2 mM L-glutamine and $200 \mathrm{mM}$ streptomycin/penicillin (Invitrogen; Thermo Fisher Scientific, Inc.) and maintained at 90-95\% relative humidity in $5 \% \mathrm{CO}_{2}$ at $37^{\circ} \mathrm{C}$. Glutamate (Sigma-Aldrich; Merck KGaA) was dissolved in water while B355252 (donated by Dr Alfred Williams; North Carolina Central University, Durham, North Carolina) was dissolved in dimethyl sulfoxide at a concentration of $10 \mathrm{mM}$. Stock solutions were diluted with cell culture medium for each experiment. Cells were subjected to glutamate stress for $18 \mathrm{~h}$ alone or after pretreatment for $2 \mathrm{~h}$ with B355252 in full culture medium. Various concentrations of glutamate $(0.25-16 \mathrm{mM})$ and B355252 $(0.16-10 \mu \mathrm{M})$ were evaluated to determine their optimal working concentrations prior to their combined use.

Cell viability assay. The viability of the HT22 cells was quantified in 96-well plates using 7-hydroxy-3H-phenoxazin-3-one 10-oxide (resazurin). A stock solution of resazurin was prepared in deionized water at a concentration of $1 \mathrm{mg} / \mathrm{ml}$. Following the aforementioned treatment of cells with glutamate and/or B355252, a $10 \mu \mathrm{l}$ aliquot of resazurin was dispensed into each well containing $100 \mu \mathrm{l}$ DMEM to achieve a final concentration of $0.1 \mathrm{mg} / \mathrm{ml}$. After $3 \mathrm{~h}$ of incubation in $5 \% \mathrm{CO}_{2}$ at $37^{\circ} \mathrm{C}$, the cells were equilibrated to room temperature for $15 \mathrm{~min}$ and the fluorescence was measured with a PHERAstar Microplate Reader (BMG Labtech GmbH) with a 540-20/590-20 nm filter. The relative fluorescence of the untreated, control cells represented $100 \%$ cell viability and the cell viability of each experimental group was converted to a percentage relative to the control.

Western blotting. For immunoblotting, treated cells were lysed in RIPA buffer (cat. no. R0278; Sigma-Aldrich; Merck KGaA) with complete protease (cat. no. P1860; Sigma-Aldrich; Merck KGaA) and phosphatase (cat. no. 52-462-51SET; MilliporeSigma) inhibitor cocktails. Following lysis, subcellular fractions of the cytosol, mitochondria and nucleus were isolated through differential centrifugation steps as previously described (18). The purity of the fractions was verified as previously reported (19). Protein concentrations were determined using the Bradford assay. Equal amounts of protein (20 $\mu \mathrm{g} /$ lane) in the total cell fractions were separated on 4-12\% NuPAGE SDS-PAGE gels (Invitrogen; Thermo Fisher Scientific, Inc.), transferred to nitrocellulose membranes, and then probed overnight at $4^{\circ} \mathrm{C}$ with the following antibodies: Phospho-(Ser616)Drp1 (p-Drp1; 1:1,000; cat. no. 3455; Cell Signaling Technology, Inc.), Drp1 (1:500; cat. no. PIPA577924; Invitrogen; Thermo Fisher Scientific, Inc.), Fis1 (1:500; cat. no. sc-98900; Santa Cruz Biotechnology, Inc.), Opal (1:1,000; cat. no. sc-30572; Santa Cruz Biotechnology, Inc.), Mfn1 (1:1,000; cat. no. sc-50330; Santa Cruz Biotechnology, Inc.), Mfn2 (1:1,000; cat. no. sc-50331; Santa Cruz Biotechnology, 
Inc.), apoptosis-inducing factor (AIF; 1:500; cat. no. sc-55519; Santa Cruz Biotechnology, Inc.), mitochondrial cytochrome $c$ oxidase subunit IV (Cox-IV; 1:1,000; cat. no. ab14744; Abcam) or $\beta$-actin (1:1,000; A1978; Sigma-Aldrich; Merck KGaA). The membranes were incubated with IRDye ${ }^{\circledR}$ 680RD goat anti-rabbit IgG (H+L) (1:2,000; cat. no. 926-68171; LI-COR Biosciences) or IRDye ${ }^{\circledR} 800 \mathrm{CW}$ goat anti-mouse IgG $(\mathrm{H}+\mathrm{L})$ (1:2,000; cat. no. 926-32210; LI-COR Biosciences) for $1 \mathrm{~h}$ at room temperature. $\beta$-actin, Cox-IV and lamin B $(1: 1,000$; cat. no. ab16048; Abcam) were used as internal loading controls. Membranes were scanned with an Odyssey LI-COR imaging system (LI-COR Biosciences). The targeting band densities were quantified using Image Studio (4.x CLx; LI-COR Biosciences) and ratios of the targeted proteins and loading control were calculated and presented.

Mitochondrial imaging. H922 cells were grown on a Lab-Tek $^{\mathrm{TM}}$ II Chamber Slide ${ }^{\mathrm{TM}}$ (Thermo Fisher Scientific, Inc.), and were treated with glutamate and/or B3552525 as aforementioned. After the treatment, the cells were labeled with MitoTracker ${ }^{\mathrm{TM}}$ Red CM-XRos (M7512; Invitrogen; Thermo Fisher Scientific, Inc.) at $37^{\circ} \mathrm{C}$ in a humidified $5 \%$ $\mathrm{CO}_{2}$ atmosphere for $30 \mathrm{~min}$. The cells were thereafter fixed with $4 \%$ paraformaldehyde in DMEM culture medium for $15 \mathrm{~min}$ at room temperature. The fixed cells were rinsed twice with PBS, mounted with Vectashield mounting medium containing DAPI (Vector Laboratories, Inc.) and analyzed using a BD Pathway ${ }^{\mathrm{TM}} 855$ High-Content Bioimager (BD Biosciences) at x100 final magnification. The experiment was repeated three times and images of a minimum of three fields per group were captured. The number of cells containing fragmented mitochondria was manually counted in each experimental condition and presented as percentage of total counted cells.

AIF immunostaining. HT22 cells were grown on Lab-Tek II Chamber Slides (cat. no. 12-565-5; Thermo Fisher Scientific, Inc.). Following $18 \mathrm{~h}$ of glutamate exposure with or without B355252 pretreatment $37^{\circ} \mathrm{C}$, the cells were fixed for $20 \mathrm{~min}$ at room temperature with $4 \%$ paraformaldehyde, washed with PBS and permeabilized in $0.3 \%$ Triton X-100 for $5 \mathrm{~min}$. The cells were blocked with $10 \%$ donkey serum $(5664605 \mathrm{ML}$; MilliporeSigma) for $1 \mathrm{~h}$ at room temperature and then incubated overnight at $4^{\circ} \mathrm{C}$ with anti-AIF primary antibody (1:200; cat. no. sc-55519). The cells were washed with PBS and then incubated for $2 \mathrm{~h}$ at room temperature with Alexa Fluor 488-conjugated secondary antibodies (1:500; cat. no. R37114; Invitrogen; Thermo Fisher Scientific, Inc.). Finally, the slides were mounted with Vectashield mounting medium containing DAPI. The slides were scanned with a BD Pathway 855 High-Content Bioimager. The experiment was repeated three times and images of a minimum of three fields per group were captured and processed for analysis.

Statistical analysis. Data are presented as the mean \pm SD of at least three independent experiments and were analyzed using Graph Pad Prism 7 software (GraphPad Software, Inc.). One-way ANOVA followed by Tukey's test was used to analyze differences among groups. $\mathrm{P}<0.05$ was considered to indicate a statistically significant difference.

\section{Results}

B355252 protects HT22 cells against glutamate-induced cell death. Concentrations of glutamate $>1.0 \mathrm{mM}$ caused significant concentration-dependent reductions in HT22 cell viability compared with that of the untreated control, with $2.0 \mathrm{mM}$ decreasing cell viability by $\sim 60 \%$ ( $\mathrm{P}<0.001$; Fig. $1 \mathrm{~A}$ and D) and higher concentrations decreasing cell viability $>90 \%$. Based on this result, $2.0 \mathrm{mM}$ glutamate was selected for subsequent experiments. B355252 alone exhibited no toxicity on HT22 cell viability at concentrations $\leq 5 \mu \mathrm{M}$ relative to the control (Fig. 1B and D). However, a 75\% loss of viability was observed when the cells were exposed to $10.0 \mu \mathrm{M}$ B355252. On the basis of these results, $2.5 \mu \mathrm{M}$ B355252 was selected for further experiments.

The pretreatment of HT22 cells with B355252 essentially prevented the toxic effect of $2.0 \mathrm{mM}$ glutamate on viability $(\mathrm{P}<0.001$; Fig. 1C and D). Notably, glutamate-induced cell death was also prevented by the simultaneous application of $5 \mu \mathrm{M} \mathrm{B} 355252$, although the level of protection was less robust compared with that of pretreatment with the compound prior to glutamate exposure (data not shown). These results demonstrate that B355252 pretreatment significantly reduced cell death and improved the survival of HT22 cells under glutamate exposure.

B355252 suppresses the glutamate-induced increase of mitochondrial fission proteins Drpl and Fis1. The exposure of HT22 cells to glutamate led to a significant 7-fold increase in the level of p-Drp1 in the mitochondrial protein fraction $(\mathrm{P}<0.001$; Fig. 2) and a 4 -fold reduction in the cytosolic level of this protein compared with the respective levels in unexposed control cells $(\mathrm{P}<0.01$; Fig. 2$)$, which suggests that p-Drp1 translocated from the cytosol to the mitochondria. Pretreatment with B355252 prior to glutamate exposure blocked the activation and translocation of Drp1 and thereby restored the balance between cytosolic and mitochondrial p-Drp1 to levels similar to those in control cells. In the mitochondrial fraction, the band densities of total Drpl relative to those of the internal loading control COX-IV were not significantly altered by the treatments. Analysis of Fis1 showed a slight but significant 1.5 -fold $(\mathrm{P}<0.01)$ upregulation of the protein in the mitochondria following glutamate exposure. Pretreatment with B355252 prevented the glutamate-dependent increase, resulting in the level of Fis1 in the cells remaining at baseline levels. No significant change in Fis1 protein was observed in the cytosolic fraction in the presence of glutamate with or without B355252. In addition, treatment of the cells with B355252 alone did not alter the levels of p-Drp1 or Fis1 in the mitochondria or cytosol of the HT22 cells.

B355252 prevents glutamate-induced changes of mitochondrial fusion markers Opal, Mfn1 and Mfn2. Western blotting revealed that B355252 caused small, statistically insignificant changes in Opa1, Mfn1 and Mfn2 protein levels in the mitochondria in comparison with those in untreated control cells, and glutamate exposure significantly increased Opa1, Mfn1 and Mfn2 expression by 40\% ( $<<0.05), 25 \%(\mathrm{P}<0.01)$ and $40 \%(\mathrm{P}<0.05)$, respectively (Fig. 3). Furthermore, B355252 
A
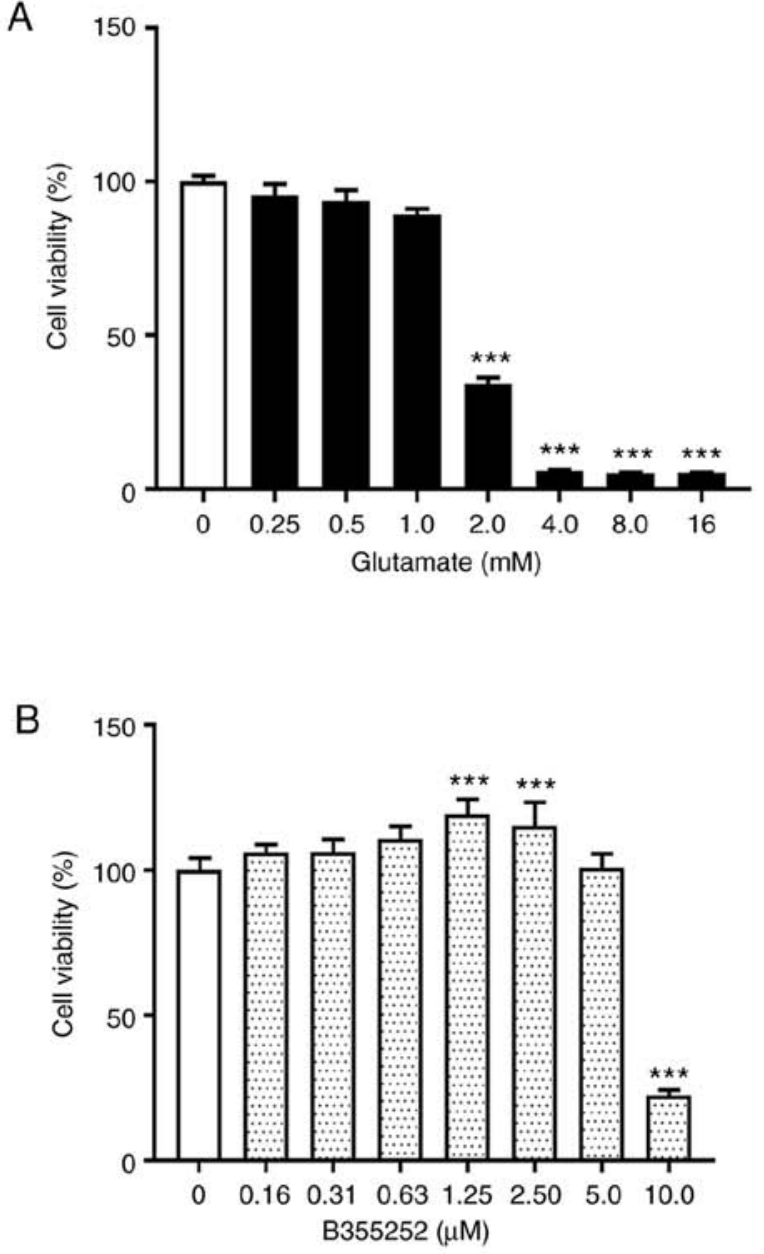

C

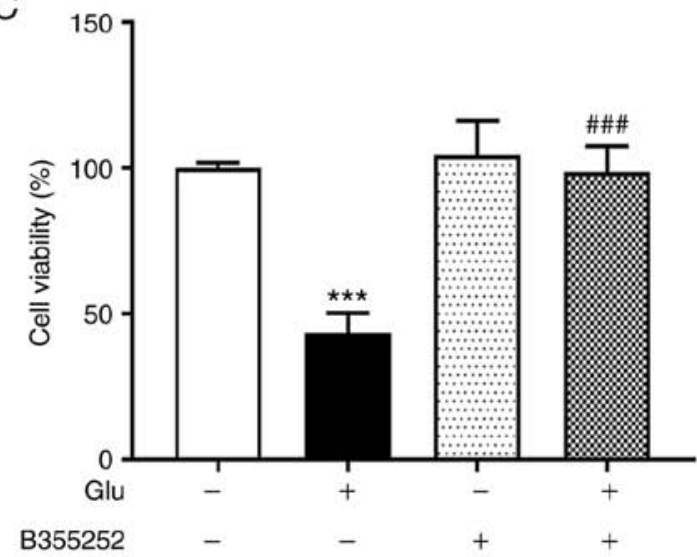

D
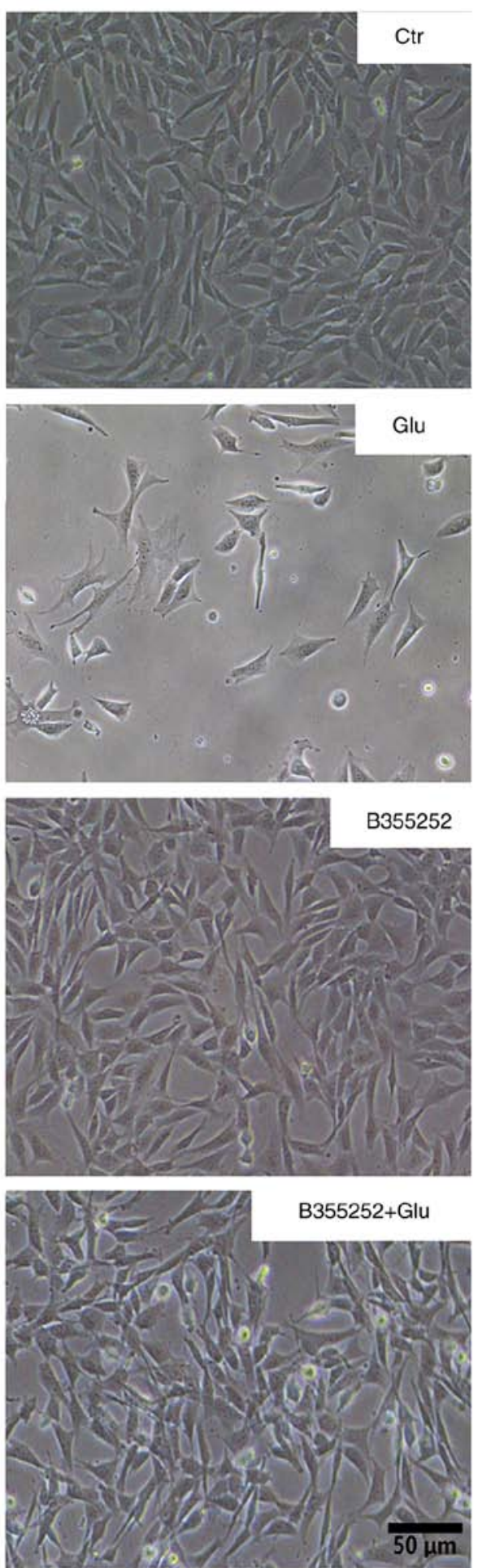

Figure 1. B355252 reduces glutamate-induced HT22 cell death. (A) HT22 cells were treated with the indicated concentrations of glutamate and cell viability was assessed with a resazurin assay. Cells exposed to glutamate concentrations $>2 \mathrm{mM}$ showed a significant reduction in cellular viability after $18 \mathrm{~h}$. Values represent the means \pm SD as a percentage of the control. ${ }^{* * *} \mathrm{P}<0.001 \mathrm{vs.} 0 \mathrm{mM}$ glutamate. (B) Concentration-dependent effect of B355252 on $\mathrm{HT} 22$ cell viability. HT22 cells tolerated B355252 well at concentrations $\leq 5 \mu \mathrm{M}$, while $10 \mu \mathrm{M}$ B355252 reduced cell viability to $\sim 25 \%$ of the control after $20 \mathrm{~h}$. ${ }^{* * *} \mathrm{P}<0.001$ vs. $0 \mu \mathrm{M}$ B355252. (C) B355252 protected cells from glutamate-induced cell death when applied $2 \mathrm{~h}$ before glutamate exposure. ${ }^{* * *} \mathrm{P}<0.001$ vs. untreated control and

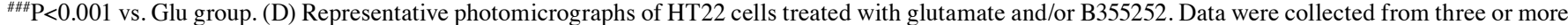
independent experiments conducted in triplicate. Values are mean \pm SD and analyzed by one-way ANOVA followed by Tukey's multiple comparison test. Glu, $2 \mathrm{mM}$ glutamate.

pretreatment significantly decreased the glutamate-induced elevations in Opal and Mfn1 proteins by $40 \%(\mathrm{P}<0.05)$ and in Mfn2 protein by $65 \%(\mathrm{P}<0.01)$ relative to the levels in cells treated with glutamate alone.
B355252 decreases glutamate-induced mitochondrial fragmentation. Based on the preceding results, morphological imaging was performed to evaluate the dynamic changes occurring in the mitochondria. Consistent with the protein 

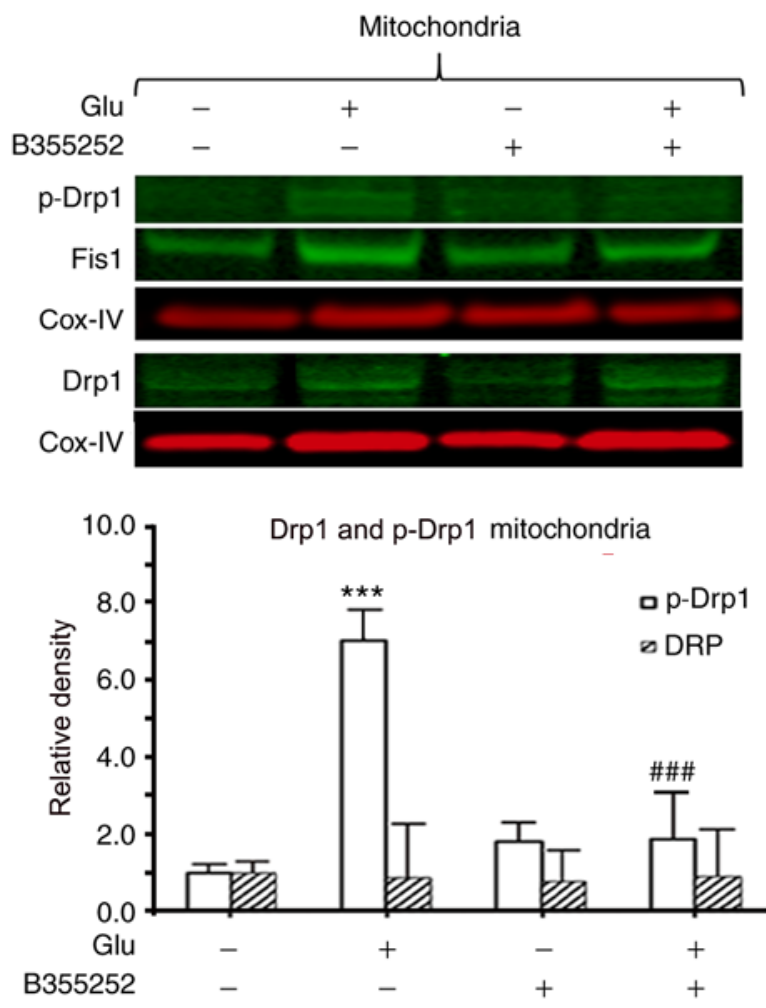

Fis1 mitochondria

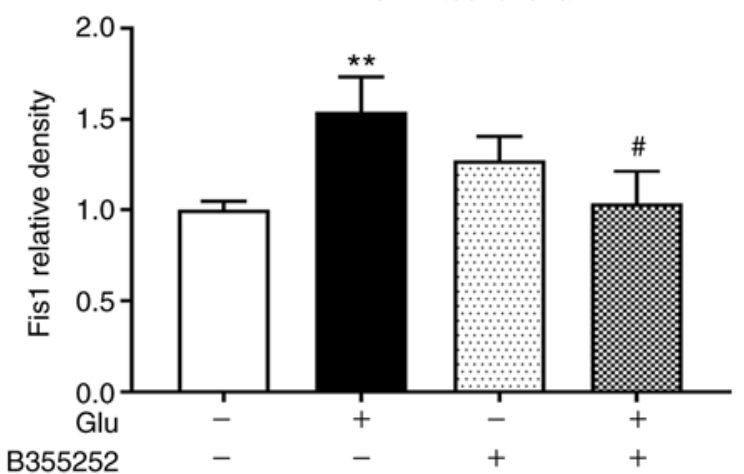

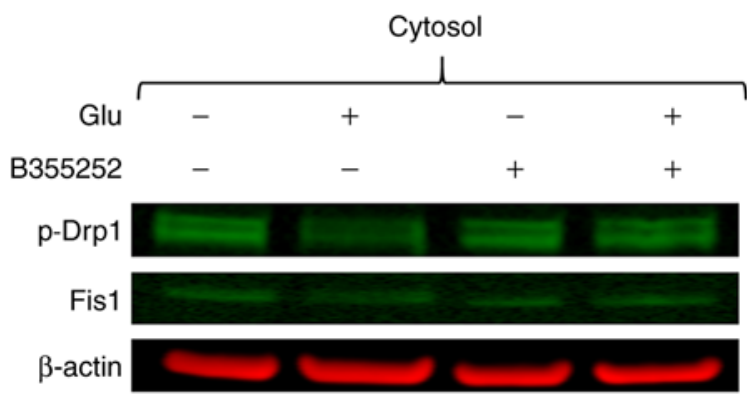

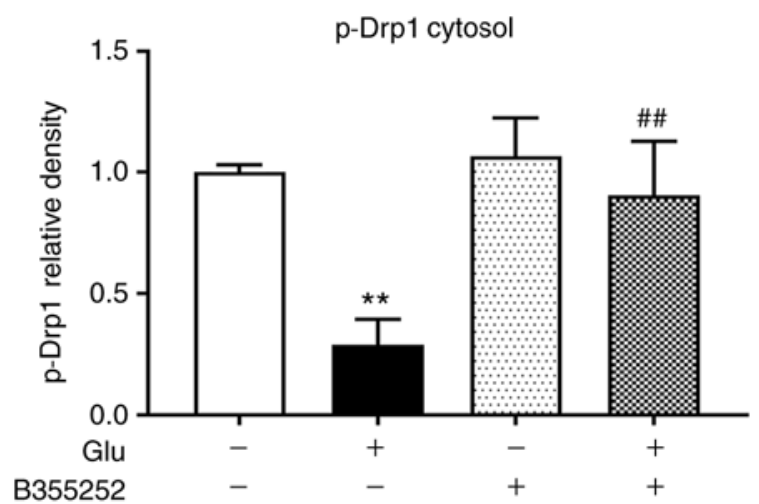

Fis1 cytosol

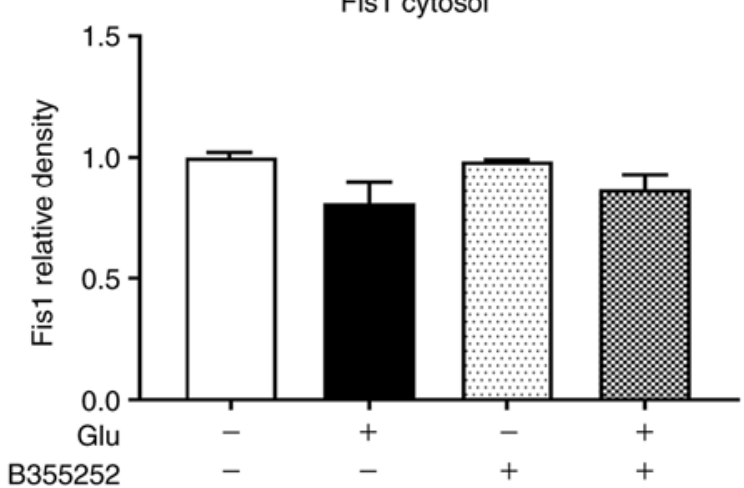

Figure 2. B355252 inhibits glutamate-induced changes in mitochondrial fission markers. Representative western blots and quantitative analysis of the protein bands of the mitochondrial fission proteins p-Drp1 and Fis1. Glutamate exposure significantly increased the levels of mitochondrial fragmentation markers p-Drp1 and Fis1 without affecting the levels of total Drp1 in the mitochondria after $18 \mathrm{~h}$. By contrast, B355252 significantly attenuated the glutamate-induced increase of p-Drp1 and Fis1 in the mitochondria of these cells. Glutamate exposure significantly reduced the cytosolic level of p-Drp1 without affecting the level of $\beta$-actin after $18 \mathrm{~h}$. B355252 attenuated the glutamate-induced reduction of p-Drp1 in the cytosol. Data are from three or more independent experiments conducted in triplicate. Values are presented as means \pm SD and were analyzed by one-way ANOVA followed by Tukey's multiple comparison test. ${ }^{* *} \mathrm{P}<0.01$

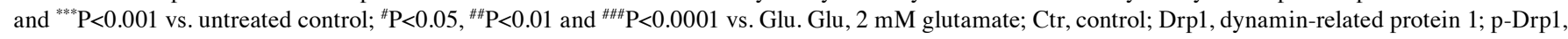
phospho-Drp1; Fis1, mitochondrial fission 1 protein; Cox-IV, mitochondrial cytochrome $c$ oxidase subunit IV.

levels of the mitochondrial fission regulators p-Drp1 and Fis1, mitochondrial imaging using MitoTracker Red revealed that marked morphological changes were induced by glutamate in the mitochondria. As seen in Fig. 4, the mitochondria in control cells displayed rod and wire shapes with a reticular mitochondrial network in the cell body. By contrast, the reticulitis of the mitochondrial network was broken into prominent small, rounded specks after glutamate exposure. However, pretreatment with B355252 protected the mitochondrial tubular network in glutamate-exposed cells, while B355252 treatment alone had no adverse effect on the HT22 cells. Counts of the numbers of cells that contain fragmented mitochondria revealed that glutamate treatment resulted in mitochondrial fragmentation in $\sim 60 \%$ of the cells, a 6 -fold increase compared with that in the control group $(\mathrm{P}<0.01)$, whereas only $30 \%$ of cells with fragmented mitochondria was observed in $\mathrm{B} 355252$ pre-treated, glutamate exposed cells. These results suggest that B355252 ameliorated glutamate-induced mitochondrial fragmentation.

B355252 suppresses the glutamate-induced elevation of proapoptotic AIF. Glutamate treatment significantly increased the AIF content in HT22 cell nuclei nearly 2-fold compared with that in the untreated control cells $(\mathrm{P}<0.05$; Fig. 5A). However, the nuclear content of AIF was markedly reduced to a level approaching that in the untreated control cells 

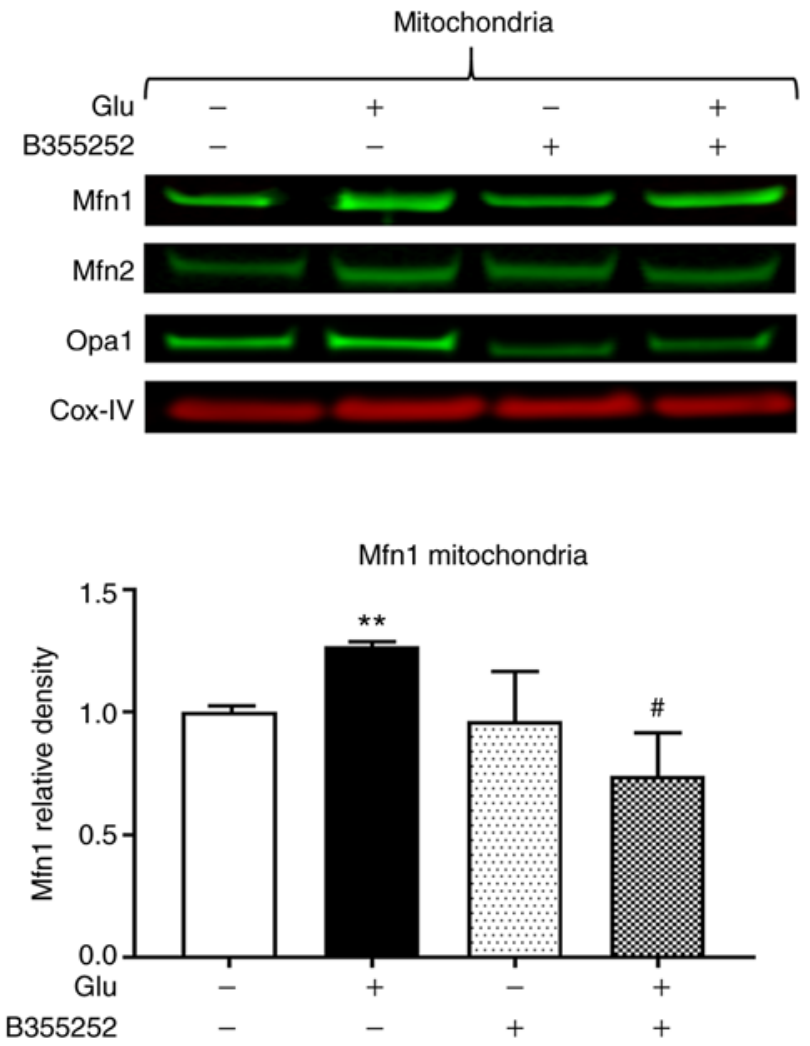

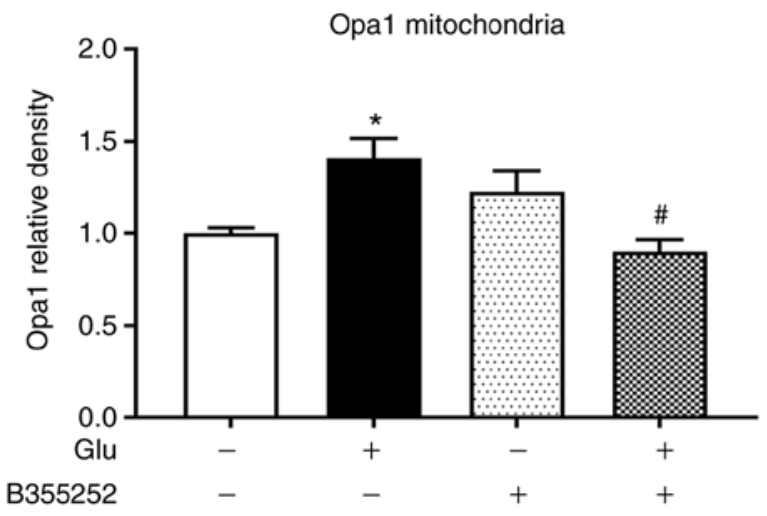

Mfn2 mitochondria

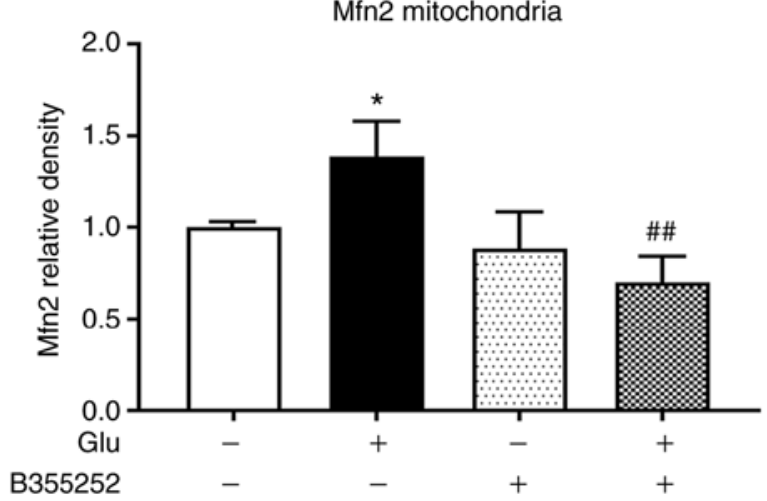

Figure 3. B355252 inhibits glutamate-induced change mitochondrial fusion markers. Representative western blots and quantitative analysis of the protein bands of the mitochondrial fusion proteins Opa1, Mfn1 and Mfn2. Glutamate exposure significantly increased the levels of the mitochondrial fusion markers Opa1, Mfn1 and Mfn2 without affecting the levels of Cox-IV after 18 h, and B355252 attenuated the glutamate-induced increase of these markers. Data are from three or more independent experiments conducted in triplicate. Values are presented as means \pm SD and were analyzed by one-way ANOVA followed by Tukey's multiple comparison test. ${ }^{*} \mathrm{P}<0.05$ and ${ }^{* *} \mathrm{P}<0.01$ vs. untreated control; ${ }^{\#} \mathrm{P}<0.05$ and ${ }^{\# \#} \mathrm{P}<0.01$ vs. Glu. Glu, 2 mM glutamate; Mfn1, mitofusin $1 ;$ Mfn2, mitofusin 2; Opa1, optic atrophy 1; Cox-IV, mitochondrial cytochrome $c$ oxidase subunit IV.
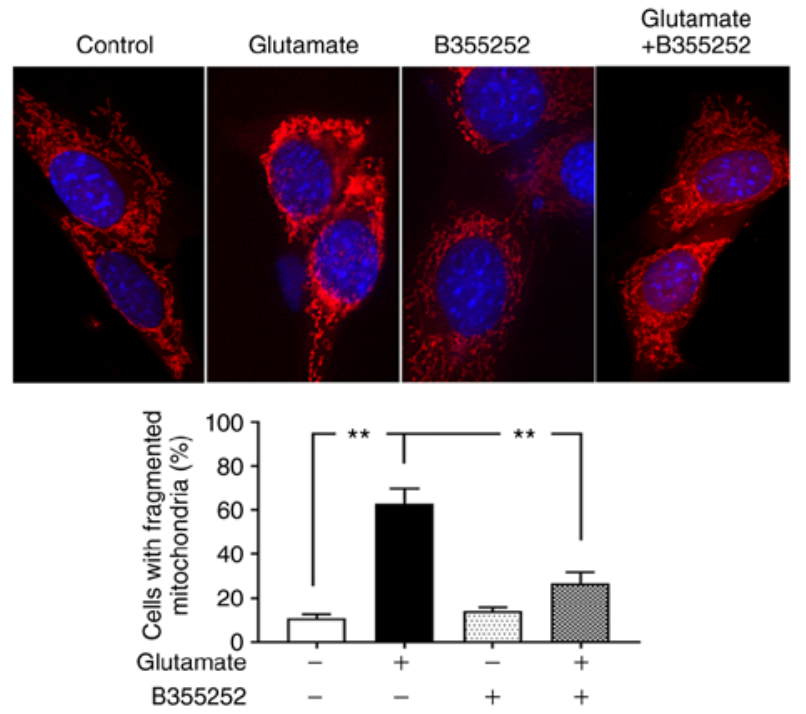

Figure 4. B355252 preserves mitochondrial morphology. Photomicrographs showing the mitochondrial morphology of HT22 cells treated with $5 \mu \mathrm{M}$ B355252 and/or $2 \mathrm{mM}$ glutamate. Mitochondrial morphology was studied using MitoTracker Red CM-XRos with a BD Pathway 855 High-Content Bioimager (final magnification, x100). Images were processed and analyzed for structural alterations. Glutamate exposure $(18 \mathrm{~h})$ disrupted the mitochondrial reticular network, resulting in small, rounded mitochondrial fragments. B355252 pretreatment attenuated the changes in the mitochondrial tubular network in glutamate-exposed cells. Data are from three or more independent experiments conducted in triplicate. Values are presented as means \pm SD and were analyzed by one-way ANOVA followed by Tukey's multiple comparison test. ${ }^{* *} \mathrm{P}<0.01$. following glutamate exposure with B355252 pretreatment; B355252 alone had no effect on the level of AIF in the nucleus. The mitochondrial content of AIF was not significantly altered by glutamate and/or B355252. These immunoblot findings were further confirmed by AIF immunocytochemistry, which showed the presence of $\sim 70 \%$ AIF positive cells following glutamate treatment, as measured by immunofluorescence localization in the nucleus. This significant increase compared with the untreated control $(\mathrm{P}<0.001)$ was significantly inhibited and maintained at control levels by pre-exposure of the cells to B355252 ( $\mathrm{P}<0.001$; Fig. 5B).

\section{Discussion}

Neurodegenerative diseases are a set of disorders that typically lead to deterioration in the function of cells of the CNS. The prevalence of these diseases is increasing owing to numerous factors, including advances in medicine that have led to increased lifespans, poor insight into the mechanisms responsible for disease initiation and progression, and the absence of effective therapies that reduce or prevent their incidence. The identification of B355252, a phenoxythiophene compound from a proprietary chemical library, as a novel agent that potentiates neurotrophin-dependent neuronal cell signaling was reported in our previous study (14-17). B355252 has been shown to exhibit a wide range of pharmacological actions, including the potentiation of NGF-initiated neurite 
A
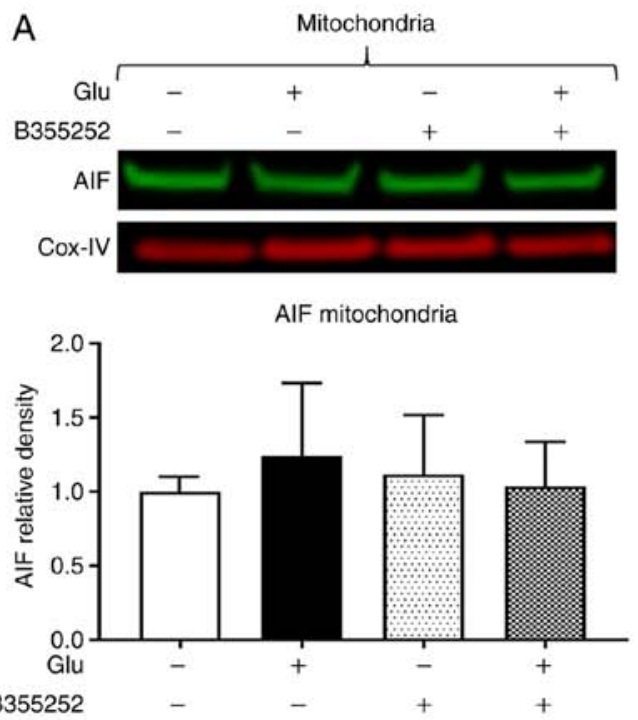

B
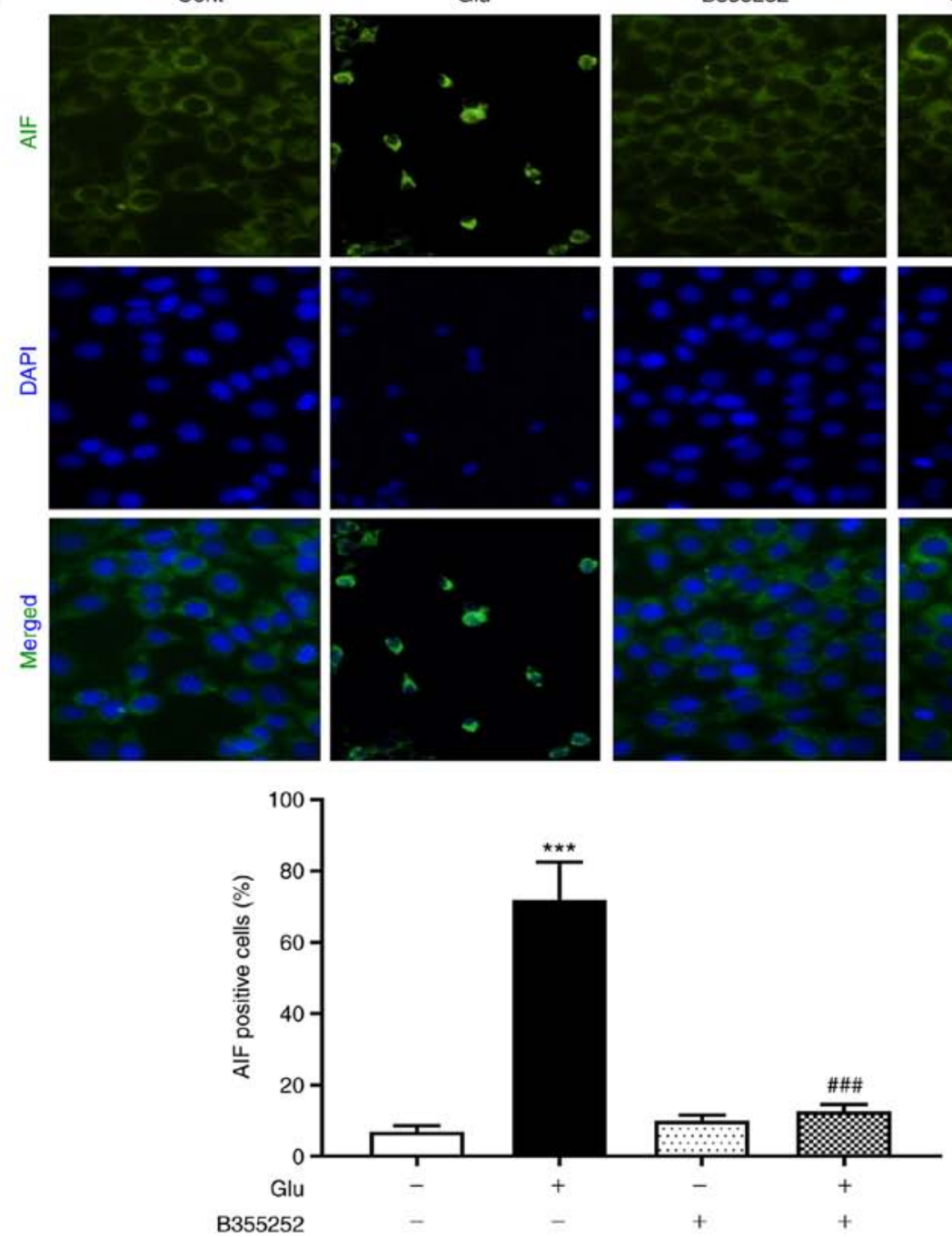

Figure 5. B355252 prevents glutamate-induced AIF release. (A) Representative western blots and quantitative analysis of the AIF protein bands in the mitochondrial and nuclear fractions of HT22 cells. Mitochondrial AIF levels were similar in the mitochondrial fractions of HT22 cells, regardless of treatment regimen. By contrast, glutamate $(2 \mathrm{mM})$ exposure significantly increased the nuclear translocation of AIF. B355252 (5 $\mu \mathrm{M})$ pretreatment blocked the nuclear translocation of AIF in cells exposed to glutamate. Values are presented as means \pm SD and were analyzed by one-way ANOVA followed by Tukey's multiple comparison tests. ${ }^{*} \mathrm{P}<0.05$ vs. untreated control; ${ }^{\#} \mathrm{P}<0.05$ vs. Glu. (B) Representative immunocytochemical photomicrographs and quantitative analysis of AIF positive cells. Perinuclear staining is observed in the control cells and cells treated with B355252 alone. The translocation of AIF to the nucleus following glutamate exposure is shown by green aggregates in the nuclei, and B355252 blocked the glutamate-induced nuclear localization of AIF. Data is representative of three or more independent experiments conducted in triplicate. Values are presented as means \pm SD and were analyzed by one-way ANOVA followed by Tukey's multiple comparison tests. ${ }^{* * *} \mathrm{P}<0.001$ vs. untreated control; ${ }^{\# \#} \mathrm{P}<0.001$ vs. Glu. Glu, glutamate; AIF, apoptosis-inducing factor; Cox-IV, mitochondrial cytochrome $c$ oxidase subunit IV. 
outgrowth and extension, and the promotion of cell survival and neuroprotection in a serum-deprivation model (15) and an in vitro model of Parkinson's disease (17). Furthermore, the rescue of neuronal cells from glutamate-induced oxidative mitochondrial injury by B355252 has been demonstrated to occur through the inhibition of ROS production and the robust blockade of $\left[\mathrm{Ca}^{2+}\right]_{\mathrm{i}}$ influx (16). In the present study, the aim was to define the role of B355252 in the attenuation of glutamate-induced injury and dysfunction of the mitochondria. The results reveal that B355252 protected HT22 neurons via the regulation of key mitochondrial remodeling proteins, preservation of the mitochondrial architecture, and prevention of the translocation of proapoptotic AIF to the nucleus.

The viability of HT22 cells was assessed using resazurin, a dye of low cytotoxicity that is reduced by viable cells to resorufin. The resazurin assay reflects mitochondrial dysfunction. Therefore, whether the decreased viability of glutamate-exposed cells and preventive effects of B355252 indicated by the resazurin assay results reflect the real cell survival status is contentious. However, images of the cells clearly show a reduction in cell density following exposure to glutamate and the restoration of cell density when B355252 was applied prior to glutamate treatment, indicating that B355252 prevented glutamate-induced cell death.

Glutamate is a major excitatory neurotransmitter in the CNS where it regulates the activity of two major classes of postsynaptic receptors, namely ionotropic and metabotropic glutamate receptors. At physiological concentrations, glutamate plays a crucial role in memory, cognition and neuronal plasticity. However, abnormally high concentrations of glutamate can lead to neuronal dysfunction, excitotoxicity and cell death, mediated in part by the overexcitation of postsynaptic glutamate receptors (20). In accordance with previous findings, the exposure of HT22 cells to increased extracellular concentrations of glutamate led to a significant concentration-dependent reduction in cell viability.

Previous studies have shown that the toxicity exerted by glutamate in HT22 cells is largely mediated via the induction of oxidative stress $(16,21)$. The formation of ROS is a consequence of glutamate-induced oxidative stress, and mitochondria are a major source of ROS in neuronal cells exposed to glutamate, acutely or chronically. ROS are produced as a normal consequence of cellular metabolism and can be harmful or beneficial, depending on homeostatic systems in the cell. However, high concentrations of glutamate can overwhelm antioxidant defense systems and result in the disruption of cellular homeostasis by increasing the accumulation of ROS, and decrease cell survival by a mechanism involving mitochondrial hyperpolarization and dysfunction $(18,22)$. It is not known whether the protective effect of B355252 against glutamate toxicity is due only to its antioxidant property. The role of B355252 in ROS, $\mathrm{Ca}^{2+}$, glutathione (GSH) and ERK kinase modulation was defined in our previous study (16). The inhibitory effect of B355252 on the glutamate-induced increases in ROS and $\mathrm{Ca}^{2+}$ levels and depletion of GSH appears similar to the antioxidant activity of N-acetylcysteine (NAC) (23). However, the changes observed using NAC, although significant, were less pronounced compared with those induced by B355252 in naïve cells. Therefore, the effectiveness of B355252 in the protection of neurons is not likely to be limited to its role as an antioxidant, and other mechanisms may be contributing to the protection conferred against glutamate-induced injury. Given that mitochondria are the major site of ROS and $\mathrm{Ca}^{2+}$ production during glutamate-dependent injury, and glutamate has been reported to affect the mitochondrial dynamics of neuronal cells $(18,24)$, the role of $\mathrm{B} 355252$ in the mitochondrial dynamics of HT22 cells was investigated in the present study. The results support the hypotheses that B355252 has a role in mitochondrial remodeling, and that an additional mechanism other than antioxidation contributes to the ability of the compound to completely protect neuronal cells from glutamate-induced stress.

The association between glutamate toxicity and mitochondrial fragmentation has previously been established. It has been reported that mitochondrial dynamic events controlled by the fission proteins Drp1 and Fis1, and the fusion proteins Opa1 and Mfn1/2, are altered during glutamate-induced stress $(18,24,25)$. Drp1, a cytoplasmic GTPase is the master regulator of mitochondrial fission. Mitochondrial fission occurs through a series of steps involving the translocation of the activated form of Drp1 from the cytoplasm to the outer mitochondrial membrane, where it drives membrane fission (12). The recruitment of Drp1 is facilitated by Fis1, a key receptor located in the outer mitochondrial membrane. Other proteins that mobilize Drp1 to the mitochondria independent of Fis1, such as Mff and the mitochondrial dynamics proteins MiD49 and MiD51, have since been identified (26,27). These proteins serve critical roles in mitochondrial fission events.

Activation of Drp1 by phosphorylation leads to division of the mitochondria to generate two daughter organelles (28). Earlier studies into the role of Drp1 showed that the overexpression of mutant Drp1 caused abnormal mitochondrial morphology in mammalian cells $(29,30)$, while the inhibition of Drp1 via the overexpression of a dominant-negative mutant prevented the loss of transmembrane potential and release of cytochrome $c$, and protected against cell death (31). Likewise, the overexpression of Fis1 causes mitochondrial fragmentation in mammalian cells, while Fis1 knockdown alleviates it $(32,33)$. Furthermore, a study of small molecule inhibitors of Drp1 activation revealed that they protected PC12 cells against ischemic neuronal injury in an oxygen-glucose deprivation model via the attenuation of mitochondrial dysfunction promoted by $\left[\mathrm{Ca}^{2+}\right]_{\mathrm{i}}$ uptake (34).

Drp1 can be phosphorylated at distinct serine residues, including Ser618 by Cdk1/cyclin B, and Ser637 or 656 by protein kinase A $(35,36)$. Previous studies have demonstrated that mitochondrial fission is promoted when Drp1 is phosphorylated at Ser616 and inhibited when phosphorylation occurs at Ser637 or Ser656 (37,38). In the present study, a significant increase in phosphorylated Drp1 levels in the mitochondria of HT22 cells was observed using a p-Drp1 (Ser616)-targeting antibody following glutamate exposure. Fis1 was also increased in the mitochondria of cells treated with glutamate, suggesting that an interaction between Drp1 and Fis1 leads to excessive mitochondrial fission and contributes to glutamate-mediated cell death. B355252 decreased the glutamate-induced increase in the levels of p-Drp1 in the mitochondria, and restored p-Drp1 levels in the cytoplasm. Therefore, these effects of B355252 support the hypothesis that the compound reduces mitochondrial fission 
by decreasing the glutamate-induced activation of Drp1 and its recruitment to the mitochondrial membrane, as well as restoring the Drp1 balance in the cytoplasm. In the present study, a 7-fold upregulation of Drp1 accompanied by increased Fis1 expression in the mitochondrial fraction was observed when cells were exposed to glutamate. These increases are consistent with the observation that the percentage of cells containing fragmented mitochondria increased significantly in glutamate-treated cells. These data suggest that glutamate activates the mitochondrial fission machinery that causes mitochondrial fragmentation and eventually cell death. The pretreatment of glutamate-exposed cells with B355252 completely blocked the glutamate-induced increases of p-Drp1 and Fis1 in the mitochondria and reduced mitochondrial fragmentation, suggesting that the protective effect of B355252 against glutamate-induced excitotoxicity is at least partially mediated through the inhibition of mitochondrial fission. One limitation of the study must be noted. Ideally the ratio of p-Drp1 to Drp1 should be calculated to evaluate whether the observed changes in p-Drp1 reflect a change in phosphorylation per se or a change of total Drp1 protein. Since total Drp1 was not detected alongside with p-Drp1 in the cytosol, whether the observed change in p-Drp1 in the cytosol reflects a change in total Drpl is not known.

Mitochondria constantly undergo cycles of fission and fusion in order to maintain the morphology and functions of the organelle (39). The fusion process is controlled by Opa1, Mfn1 and Mfn2, three large GTPases belonging to the dynamin family of proteins. Mfn1 and Mfn2 are located on the outer mitochondrial membrane and are key regulators of outer membrane fusion, while Opa1 is embedded in the inner membrane and is involved in inner mitochondrial membrane fusion (40). Several lines of evidence demonstrate that mitochondrial fusion serves an essential role in mammalian development $(41,42)$. Opa1 is a key regulator of neuronal fate during excitotoxic cell death $(43,44)$. The association of glutamate-induced mitochondrial fragmentation with reduced levels of mitochondrial fusion proteins has been observed in various studies. For example, glutamate toxicity reduces the levels of Opa1 and promotes neuronal cell death by inducing mitochondrial morphology defects, whereas the upregulation of Opal confers significant protection against $\mathrm{N}$-methyl-D-aspartate-induced neuronal death (45). Studies have also shown that Mfn2 deficiency leads to mitochondrial fragmentation and neuronal death, and renders motor neurons vulnerable to glutamate excitotoxicity, while the overexpression of Mfn2 prevents glutamate-induced fragmentation of the mitochondria, and blocks the death of spinal cord motor neurons and cortical neurons in vitro and in vivo (46-50). However, in the present study, 0.25- to 0.4-fold increases in the levels of the pro-fusion proteins Opal and Mfn1/2 were observed in the mitochondrial fraction after glutamate incubation. This could be a protective reaction of the mitochondria to glutamate stress, similar to the expression of certain pro-survival genes following glutamate or ischemic stress. Nevertheless, even with the slight increases of pro-fusion proteins, the mitochondria were destined for fragmentation, as evidenced by mitochondrial imaging, suggesting that glutamate predominantly induced mitochondrial fission even in the presence of slightly elevated levels of fusion proteins.
Pretreatment with B355252 prevented the increases in mitochondrial Opa1 and Mfn1/2 levels, in addition to blocking the glutamate-induced increases p-Drp1 and Fis1, suggesting that maintenance of the mitochondrial dynamic balance plays a key role in the rescue of the cells by B355252.

A direct consequence of glutamate-induced toxicity in cells is bioenergetic failure provoked by collapse of the mitochondrial membrane potential. Dissipation of the membrane potential triggers prolonged opening of mitochondrial permeability transition pores (MPTPs) under the control of cyclophilin-D. The formation of MPTPs leads to permeabilization of the mitochondria and the influx of molecules into the mitochondrial matrix, which in turn causes the mitochondria to swell and rupture, releasing several apoptotic factors that promote cell death (51). AIF, a key signaling molecule of the caspase-independent apoptotic pathway, is localized in the mitochondrial intermembrane space. AIF is released into the cytoplasm in response to mitochondrial damage, where its interaction with pro-death protein partners, such as cyclophilin A, initiates translocation into the nucleus, leading to DNA fragmentation (52). Previous studies have demonstrated the neuroprotective effects of AIF inhibition in vitro and in vivo $(53,54)$. The results of the present study confirmed previous observations of glutamate-mediated increases in the transfer of AIF from the mitochondria to the nucleus in HT22 cells (55). Immunocytochemical staining revealed a high number of AIF positive cells under acute glutamate challenge. B355252 abrogated the nuclear translocation of AIF, and was neuroprotective when applied prior to glutamate insult.

In conclusion, the phenoxythiophene compound B355252 conferred neuroprotective effects on HT22 cells in vitro by attenuating glutamate-induced dysfunction of the mitochondrial fission process. The glutamate-induced upregulation of Drp1 and Fis1 was effectively blocked by B355252. In contrast with previous studies, glutamate increased, rather than inhibited Opa1 and Mfn1/2 expression to promote mitochondrial dysfunction in the HT22 cell model. B355252 inhibited the glutamate-induced effects on these proteins and protected the cells against mitochondrial dysfunction. In addition, B355252 prevented the translocation of AIF from the mitochondria to the nucleus. These results suggest that B355252 exerts its neuroprotective effects through mitochondria-dependent events. However, the molecular targets of B355252 and its mechanisms of action remain to be elucidated.

\section{Acknowledgements}

The authors greatly appreciate Dr Hernán Navarro from North Carolina Central University (Durham, North Carolina) for proofreading and English editing.

\section{Funding}

$\mathrm{YZ}$ is supported by the Project of Innovation and Entrepreneurship for Overseas Students of Ningxia Hui Autonomous Region (2018) and Ningxia Natural Science Foundation (grant no. 2019AAC03236). Biomanufacturing Research Institute and Technology Enterprise is partially funded by the Golden Leaf Foundation. 


\section{Availability of data and materials}

The datasets used and/or analyzed during the current study are available from the corresponding author on reasonable request.

\section{Authors' contributions}

PAL and GI conceived and designed the experiments. YZ and NSG performed the experiments. YZ and PAL analyzed the data. YZ, PAL and GI wrote the manuscript. YZ, GI and PAL have confirmed the authenticity of the raw data. All authors read and approved the final manuscript.

\section{Ethics approval and consent to participate}

Not applicable.

\section{Patient consent for publication}

Not applicable.

\section{Competing interests}

The authors declare that they have no competing interests.

\section{References}

1. Fernandes D and Carvalho AL: Mechanisms of homeostatic plasticity in the excitatory synapse. J Neurochem 139: 973-996, 2016.

2. Dzubay JA and Jahr CE: The concentration of synaptically released glutamate outside of the climbing fiber-Purkinje cell synaptic cleft. J Neurosci 19: 5265-5274, 1999.

3. Choi DW: Glutamate neurotoxicity and diseases of the nervous system. Neuron 1: 623-634, 1988.

4. Lau A and Tymianski M: Glutamate receptors, neurotoxicity and neurodegeneration. Pflugers Arch 460: 525-542, 2010.

5. Lewerenz J and Maher P: Chronic glutamate toxicity in neurodegenerative diseases-what is the evidence? Front Neurosci 9: 469, 2015.

6. Mehta A, Prabhakar M, Kumar P, Deshmukh R and Sharma PL: Excitotoxicity: Bridge to various triggers in neurodegenerative disorders. Eur J Pharmacol 698: 6-18, 2013.

7. Jezek J, Cooper KF and Strich R: Reactive oxygen species and mitochondrial dynamics: The Yin and Yang of mitochondrial dysfunction and cancer progression. Antioxidants (Basel) 7: 13 2018.

8. Bock FJ and Tait SWG: Mitochondria as multifaceted regulators of cell death. Nat Rev Mol Cell Biol 21: 85-100, 2020.

9. Vakifahmetoglu-Norberg H, Ouchida AT and Norberg E: The role of mitochondria in metabolism and cell death. Biochem Biophys Res Commun 482: 426-431, 2017.

10. Archer SL: Mitochondrial dynamics-mitochondrial fission and fusion in human diseases. N Engl J Med 369: 2236-2251, 2013.

11. Westermann B: Mitochondrial fusion and fission in cell life and death. Nat Rev Mol Cell Biol 11: 872-884, 2010.

12. Tandler B, Hoppel CL and Mears JA: Morphological pathways of mitochondrial division. Antioxidants (Basel) 7: 30, 2018.

13. Song Z, Ghochani M, McCaffery JM, Frey TG and Chan DC: Mitofusins and OPA1 mediate sequential steps in mitochondrial membrane fusion. Mol Biol Cell 20: 3525-3532, 2009.

14. Yeyeodu ST, Witherspoon SM, Gilyazova N and Ibeanu GC: A rapid, inexpensive high throughput screen method for neurite outgrowth. Curr Chem Genomics 4: 74-83, 2010.

15. Williams AL, Dandepally SR, Gilyazova N, Witherspoon SM and Ibeanu G: Microwave-assisted synthesis of 4-chloro-N-(naphtha len-1-ylmethyl)-5-(3-(piperazin-1-yl)phenoxy)thiophene-2-sulfo namide (B-355252): A new potentiator of Nerve Growth Factor (NGF)-induced neurite outgrowth. Tetrahedron 66: 9577-9581, 2010 .
16. Gliyazova NS, Huh EY and Ibeanu GC: A novel phenoxy thiophene sulphonamide molecule protects against glutamate evoked oxidative injury in a neuronal cell model. BMC Neurosci 14: 93, 2013.

17. Gliyazova NS and Ibeanu GC: The chemical molecule B355252 is neuroprotective in an in vitro model of Parkinson's disease. Cell Mol Neurobiol 36: 109-122, 2016.

18. Kumari S, Mehta SL and Li PA: Glutamate induces mitochondrial dynamic imbalance and autophagy activation: Preventive effects of selenium. PLoS One 7: e39382, 2012.

19. Mendelev N, Mehta SL, Witherspoon S, He Q, Sexton JZ and Li PA: Upregulation of human selenoprotein $\mathrm{H}$ in murine hippocampal neuronal cells promotes mitochondrial biogenesis and functional performance. Mitochondrion 11: 76-82, 2011.

20. Sattler R and Tymianski M: Molecular mechanisms of glutamate receptor-mediated excitotoxic neuronal cell death. Mol Neurobiol 24: 107-129, 2001.

21. Stanciu M, Wang Y, Kentor R, Burke N, Watkins S, Kress G, Reynolds I, Klann E, Angiolieri MR, Johnson JW and DeFranco DB: Persistent activation of ERK contributes to glutamate-induced oxidative toxicity in a neuronal cell line and primary cortical neuron cultures. J Biol Chem 275: 12200-12206, 2000.

22. Kang Y, Tiziani S, Park G, Kaul M and Paternostro G: Cellular protection using Flt3 and PI3Kalpha inhibitors demonstrates multiple mechanisms of oxidative glutamate toxicity. Nat Commun 5: 3672, 2014.

23. Bavarsad Shahripour R, Harrigan MR and Alexandrov AV: $\mathrm{N}$-acetylcysteine (NAC) in neurological disorders: Mechanisms of action and therapeutic opportunities. Brain Behav 4: 108-122, 2014.

24. Ma YM, Ibeanu G, Wang LY, Zhang JZ, Chang Y, Dong JD, Li PA and Jing L: Selenium suppresses glutamate-induced cell death and prevents mitochondrial morphological dynamic alterations in hippocampal HT22 neuronal cells. BMC Neurosci 18: $15,2017$.

25. Sanderson TH, Raghunayakula S and Kumar R: Release of mitochondrial Opal following oxidative stress in HT22 cells. Mol Cell Neurosci 64: 116-122, 2015.

26. Otera H, Wang C, Cleland MM, Setoguchi K, Yokota S, Youle RJ and Mihara K: Mff is an essential factor for mitochondrial recruitment of Drp1 during mitochondrial fission in mammalian cells. J Cell Biol 91: 1141-1158, 2010.

27. Palmer CS, Osellame LD, Laine D, Koutsopoulos OS, Frazier AE and Ryan MT: MiD49 and MiD51, new components of the mitochondrial fission machinery. EMBO Rep 12: 565-573, 2011.

28. Rosenbloom AB, Lee SH, To M, Lee A, Shin JY and Bustamante C: Optimized two-color super resolution imaging of Drp1 during mitochondrial fission with a slow-switching Dronpa variant. Proc Natl Acad Sci USA 111: 13093-13098, 2014.

29. Smirnova E, Griparic L, Shurland DL and van der Bliek AM: Dynamin-related protein Drp1 is required for mitochondrial division in mammalian cells. Mol Biol Cell 12: 2245-2256, 2001.

30. Smirnova E, Shurland DL, Ryazantsev SN and van der Bliek AM: A human dynamin-related protein controls the distribution of mitochondria. J Cell Biol 143: 351-358, 1998.

31. Frank S, Gaume B, Bergmann-Leitner ES, Leitner WW, Robert EG, Catez F, Smith CL and Youle RJ: The role of dynamin-related protein 1, a mediator of mitochondrial fission, in apoptosis. Dev Cell 1: 515-525, 2001.

32. Stojanovski D, Koutsopoulos OS, Okamoto K and Ryan MT: Levels of human Fis1 at the mitochondrial outer membrane regulate mitochondrial morphology. J Cell Sci 117: 1201-1210, 2004.

33. James DI, Parone PA, Mattenberger Y and Martinou JC: hFis1, a novel component of the mammalian mitochondrial fission machinery. J Biol Chem 278: 36373-36379, 2003.

34. Tian Y, Li B, Shi WZ, Chang MZ, Zhang GJ, Di ZL and Liu Y: Dynamin-related protein 1 inhibitors protect against ischemic toxicity through attenuating mitochondrial $\mathrm{Ca} 2+$ uptake from endoplasmic reticulum store in PC12 cells. Int J Mol Sci 15: 3172-3185, 2014

35. Cribbs JT and Strack S: Reversible phosphorylation of Drp1 by cyclic AMP-dependent protein kinase and calcineurin regulates mitochondrial fission and cell death. EMBO Rep 8: 939-944, 2007.

36. Chang CR and Blackstone C: Cyclic AMP-dependent protein kinase phosphorylation of Drp1 regulates its GTPase activity and mitochondrial morphology. J Biol Chem 282: 21583-21587, 2007.

37. Taguchi N, Ishihara N, Jofuku A, Oka T and Mihara K: Mitotic phosphorylation of dynamin-related GTPase Drp1 participates in mitochondrial fission. J Biol Chem 282: 11521-11529, 2007. 
38. Otera $\mathrm{H}$ and Mihara $\mathrm{K}$ : Molecular mechanisms and physiologic functions of mitochondrial dynamics. J Biochem 149: 241-251, 2011.

39. Bartolák-Suki E, Imsirovic J, Nishibori Y, Krishnan R and Suki B: Regulation of mitochondrial structure and dynamics by the cytoskeleton and mechanical factors. Int J Mol Sci 18: 1812, 2017.

40. Meeusen S, McCaffery JM and Nunnari J: Mitochondrial fusion intermediates revealed in vitro. Science 305: 1747-1752, 2004.

41. Wai T, García-Prieto J, Baker MJ, Merkwirth C, Benit P, Rustin P, Rupérez FJ, Barbas C, Ibañez B and Langer T: Imbalanced OPA1 processing and mitochondrial fragmentation cause heart failure in mice. Science 350: aad0116, 2015.

42. Bertholet AM, Delerue T, Millet AM, Moulis MF, David C, Daloyau M, Arnauné-Pelloquin L, Davezac N, Mils V, Miquel MC, et al: Mitochondrial fusion/fission dynamics in neurodegeneration and neuronal plasticity. Neurobiol Dis 90: 3-19, 2016.

43. Kushnareva YE, Gerencser AA, Bossy B, Ju WK, White AD, Waggoner J, Ellisman MH, Perkins G and Bossy-Wetzel E: Loss of OPA1 disturbs cellular calcium homeostasis and sensitizes for excitotoxicity. Cell Death Differ 20: 353-365, 2013.

44. Nguyen D, Alavi MV, Kim KY, Kang T, Scott RT, Noh YH, Lindsey JD, Wissinger B, Ellisman MH, Weinreb RN, et al: A new vicious cycle involving glutamate excitotoxicity, oxidative stress and mitochondrial dynamics. Cell Death Dis 2: e240, 2011

45. Jahani-Asl A, Poilon-Larose K, Xu W, Maclaurin JG, Park DS, McBride HM and Slack RS: The mitochondrial inner membrane GTPase, optic atrophy 1 (Opa1), restores mitochondrial morphology and promotes neuronal survival following excitotoxicity. J Biol Chem 286: 4772-4782, 2011.

46. Wang W, Zhang F, Li L, Tang F, Siedlak SL, Fujioka H, Liu Y, $\mathrm{Su} \mathrm{B}, \mathrm{Pi}$ Y and Wang X: MFN2 couples glutamate excitotoxicity and mitochondrial dysfunction in motor neurons. J Biol Chem 290: 168-182, 2015.

47. Wang X, Su B, Siedlak SL, Moreira PI, Fujioka H, Wang Y, Casadesus G and Zhu X: Amyloid-beta overproduction causes abnormal mitochondrial dynamics via differential modulation of mitochondrial fission/fusion proteins. Proc Natl Acad Sci USA 105: 19318-19323, 2008.
48. Jahani-Asl A, Vheung EC, Neuspiel M, MacLaurin JG, Fortin A, Park DS, McBride HM and Slack RS: Mitofusin 2 protects cerebellar granule neurons against injury-induced cell death. J Biol Chem 282: 23788-23798, 2007.

49. Neuspiel M,Zunino R, Gangaraju S, Rippstein P and McBride H: Activated mitofusin 2 signals mitochondrial fusion, interferes with Bax activation, and reduces susceptibility to radical induced depolarization. J Biol Chem 280: 25060-25070, 2005.

50. Ju Wk, Lindsey JD, Angert M, Patel A and Weinreb RN: Glutamate receptor activation triggers OPA1 release and induces apoptotic cell death in ischemic rat retina. Mol Vis 14: 2629-2638, 2008.

51. Halestrap AP: What is the mitochondrial permeability transition pore? J Mol Cell Cardiol 46: 821-831, 2009.

52. Sevrioukova IF: Apoptosis-inducing factor: Structure, function, and redox regulation. Antioxid Redox Signal 14: 2545-2579, 2011.

53. Jantas D, Greda A, Leskiewicz M, Grygier B, Pilc A and Lason W: Neuroprotective effects of mGluR II and III activators against staurosporine- and doxorubicin-induced cellular injury in SH-SY5Y cells: New evidence for a mechanism involving inhibition of AIF translocation. Neurochem Int 88: 124-137, 2015.

54. Piao CS, Loane DJ, Stoica BA, Li S, Hanscom M, Cabatbat R, Blomgren K and Faden AI: Combined inhibition of cell death induced by apoptosis inducing factor and caspases provides additive neuroprotection in experimental traumatic brain injury. Neurobiol Dis 46: 745-758, 2012.

55. Tobaben S, Grohm J, Seiler A, Conrad M, Plesnila N and Culmsee C: Bid-mediated mitochondrial damage is a key mechanism in glutamate-induced oxidative stress and AIF-dependent cell death in immortalized HT-22 hippocampal neurons. Cell Death Differ 18: 282-292, 2011.

This work is licensed under a Creative Commons Attribution-NonCommercial-NoDerivatives 4.0 International (CC BY-NC-ND 4.0) License. 